\title{
New HST/ACS Observations of Dwarf Star-Forming Galaxies with Extreme Properties of Cosmological Relevance
}

\author{
F. Annibali ${ }^{1}$, A. Aloisi ${ }^{2}$, R. Van der Marel $^{1}$, J. Mack$^{1}$, and M. Tosi ${ }^{3}$ \\ ${ }^{1}$ Space Telescope Science Institute, Baltimore, USA \\ ${ }^{2}$ ESA, Baltimore, USA \\ ${ }^{3}$ INAF-Osservatorio Astronomico di Bologna, Bologna, Italy
}

\begin{abstract}
We present deep Color Magnitude Diagrams (CMDs), based on HST/ACS data, of two star-forming dwarf galaxies, NGC4449 and IZw18.
\end{abstract}

Keywords. galaxies: evolution; galaxies: stellar content; galaxies: individual (NGC449, IZw18).

We present the first results of two ongoing HST/ACS programs (\#10585 and \#10586, PI Aloisi) aimed at studying the evolution of two nearby dwarf galaxies, namely NGC4449 and IZw18. New deep broad band observations have been acquired in order to resolve the galaxies into single stars and infer their Star Formation History (SFH) through the analysis of the CMDs.

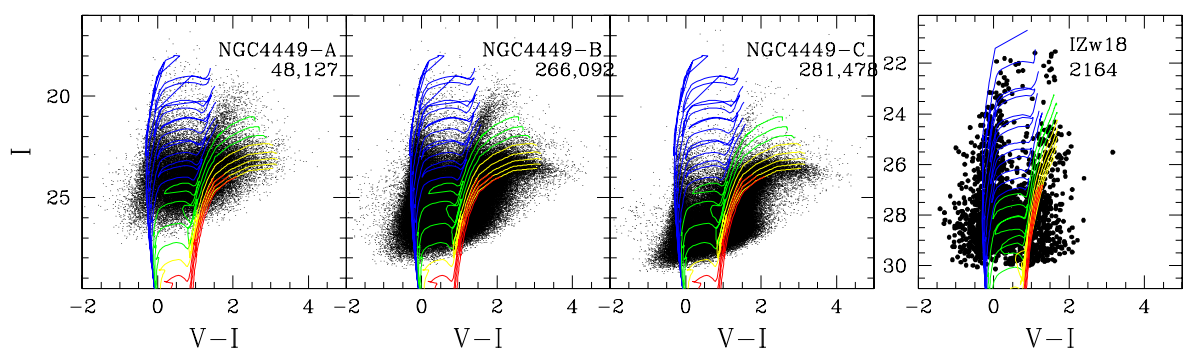

Figure 1. Left panels: I, V-I CMD for three regions of NGC4449 of increasing galacto-centric distance (from A to C). On the observed CMDs we have superimposed the Padova isochrones at $Z=0.004$ and for ages in the range (6 Myr - $10 \mathrm{Gyr}$ ). Right panel: CMD of IZw18 (main + secondary body) with Padova isochrones at $Z=0.0004$.

NGC4449 is a global starburst with a widespread SF activity that could have been triggered by merging or accretion. We show in Figure 1 the I, V-I CMD for three separate regions of increasing galacto-centric distance. The total number of photometred stars is 595,697 . The CMDs show a clear gradient in the stellar population, with the most young massive stars located towards the center. We also find that the Red Giant Branch (RGB) in the external field is $\sim 0.1$ mag redder than in the intermediate regions, again suggesting a younger population in the center.

$\underline{\mathrm{IZw} 18}$ is a Blue compact Dwarf (BCD) Galaxy with the lowest nebular netallicity of all known nearby galaxies, and it is also the most controversial case of a potentially young galaxy in the Local Universe. Our HST/ACS data yeld a deep CMD for the resolved stars for the main plus secondary body (see right panel of Figure 1). The CMD shows that faint red stars, potentially in the RGB phase (ages $>1$ Gyr), are present in IZw18. 